\title{
Thermal multicomponent lattice Boltzmann model for catalytic reactive flows
}

\author{
Jinfen Kang, ${ }^{*}$ Nikolaos I. Prasianakis, ${ }^{\dagger}$ and John Mantzaras ${ }^{\ddagger}$ \\ Combustion Research Laboratory Paul Scherrer Institute, CH-5232 Villigen PSI, Switzerland \\ (Received 10 February 2014; revised manuscript received 16 May 2014; published 30 June 2014)
}

\begin{abstract}
Catalytic reactions are of great interest in many applications related to power generation, fuel reforming and pollutant abatement, as well as in various biochemical processes. A recently proposed lattice Boltzmann model for thermal binary-mixture gas flows [J. Kang, N. I. Prasianakis, and J. Mantzaras, Phys. Rev. E. 87, 053304 (2013)] is revisited and extended for the simulation of multispecies flows with catalytic reactions. The resulting model can handle flows with large temperature and concentration gradients. The developed model is presented in detail and validated against a finite volume Navier-Stokes solver in the case of channel-flow methane catalytic combustion. The surface chemistry is treated with a one-step global reaction for the catalytic total oxidation of methane on platinum. In order to take into account thermal effects, the catalytic boundary condition of S. Arcidiacono, J. Mantzaras, and I. V. Karlin [Phys. Rev. E 78, 046711 (2008)] is adapted to account for temperature variations. Speed of sound simulations further demonstrate the physical integrity and unique features of the model.
\end{abstract}

DOI: 10.1103/PhysRevE.89.063310

PACS number(s): 47.11.- j, 47.70.-n, 05.20.-y

\section{INTRODUCTION}

The lattice Boltzmann (LB) method has become a very popular platform for the simulation of complex flow physics that range from laminar and turbulent flows to multiphase and particle or porous media flows [1-9]. For the construction of a mixture model, it is required to control the viscous dynamics as well as the molecular diffusion of each species through the collision process in an efficient way. This usually leads to two or more relaxation parameter collision submodels, such that different approaches have produced many nonthermal multicomponent LB models [10-17]. Regarding the modeling that takes into account temperature dynamics (thermal LB), a thermal binary mixture model has recently been proposed on standard lattices [18], while theoretical aspects dealing with thermal multicomponent flows on extended lattices were reported in Ref. [19].

Catalytic reactions are of key importance in many applications related to power generation, fuel reforming, pollutant abatement, and various biochemical processes [20]. However, the LB-relevant catalytic studies presented in the literature are mainly focused on isothermal flows [21-24]. In energy conversion systems, such as catalytic combustion reactors or solid oxide fuel cells, the reactive flows are subject to large temperature and concentration variations. Consequently modeling of such flows requires the correct description of mass, momentum, and heat (thermal energy) transport.

In this paper the lattice Boltzmann model for thermal binary-mixture gaseous nonreactive flows presented in Ref. [18] is extended to simulate multicomponent (three or more species) flows with catalytic reactions. In contrast to the thermal binary-mixture model, each species has its own characteristic relaxation time. This allows direct control of the transport properties of each species (viscosity, diffusivity, conductivity), resulting in a realistic description of mixture flows. During the theoretical derivation, all existing deviation terms

\footnotetext{
*kangjinfen@gmail.com

${ }^{\dagger}$ Corresponding author: nikolaos.prasianakis@psi.ch

${ }_{\ddagger}^{\dagger}$ ioannis.mantzaras@psi.ch
}

are identified and neutralized in order to guarantee the correct thermo-hydrodynamic limits. The theoretical derivation is followed by numerical validations. An interesting feature of this model is highlighted by studying the speed of sound, which reveals the potential of the model to describe acoustics. The speed of sound of the gaseous mixture is computed at different temperatures and compositions, and the results are validated against theoretical values.

The proposed model is further validated in thermal reactive flows in a catalytic channel. For this application, a thermal surface reactive boundary condition is required to describe the catalytic reaction mechanism, which takes into account the temperature information. The catalytic diffusive boundary condition [21,25] is hence adapted to include temperature description. The flow under consideration is the catalytic oxidation of methane, whereby the surface chemistry is treated by using a one-step global reaction mechanism for the total oxidation of methane on platinum [26]. The incoming methane-air mixture is fully premixed, while the channel wall temperature is kept constant. For different flow conditions, i.e., inlet velocity and inlet temperature, the LB simulation results are compared against a finite volume Navier-Stokes solver [27].

This article is organized as follows. In Sec. II the kinetic model for thermal multicomponent flows is introduced. In Sec. III, asymptotic analysis of the model behavior is examined, and the introduction of correction terms leads to the recovery of the correct macroscopic limit and full control of the transport properties. In Sec. IV the physical integrity of the model is tested by measuring the speed of sound for different mixture conditions. In Sec. V the catalytic diffusive boundary condition of Ref. [21,25] is extended to include temperature information in addition to surface chemistry. Simulation results and comparison with a well-established Navier-Stokes solver are presented for the flow in a catalytic channel with large temperature variations.

\section{KINETIC MODEL FOR THERMAL MULTICOMPONENT FLOWS}

The thermal multicomponent model kinetic equation is extended from the thermal binary-mixture model of Ref. [18]. 
For a multicomponent mixture flow composed of $N$ species, each species is assigned with a one-particle distribution function, evolving with its own kinetic equation and carrying its density, momentum, and energy information in a consistent way. The kinetic equation for each species is

$$
\begin{aligned}
\partial_{t} f_{j i}+c_{j i \alpha} \partial_{\alpha} f_{j i}= & -\frac{1}{\tau_{j 1}}\left(f_{j i}-f_{j i}^{*}\right)-\frac{1}{\tau_{j 2}}\left(f_{j i}^{*}-f_{j i}^{\mathrm{eq}}\right) \\
& +\psi_{j i}+\phi_{j i}, \quad j=1,2, \ldots, N,
\end{aligned}
$$

where $i=0, \ldots, 8$ is the index of discrete velocities $c_{j i \alpha}$, with $\alpha$ being the coordinate index $(\{\alpha=x, y, z\})$. The current model employs a standard two-dimensional nine-velocity lattice (D2Q9) for the spatial and velocity discretization, with discrete velocities written as

$$
\begin{aligned}
& c_{j i x}=c_{j}\{0,1,0,-1,0,1,-1,-1,1\}, \\
& c_{j i y}=c_{j}\{0,0,1,0,-1,1,1,-1,-1\},
\end{aligned}
$$

with $c_{j}=\sqrt{1 / M_{j}}$ and $M_{j}$ the lattice speed and the molar mass of species $j$, respectively. All species are solved on the same grid system by setting the lightest species to propagate onlattice and all other heavier off-lattice species are interpolated to the common grid. Extension of the current model to threedimensional 27 velocity lattice (D3Q27) is straightforward.

The BGK relaxation process is split in two parts $[18,28$ 31]. The first part is characterized by a relaxation toward an auxiliary state, represented by $f_{j i}^{*}$, with characteristic time $\tau_{j 1}$; the second part describes relaxation toward the thermodynamic equilibrium $f_{j i}^{\mathrm{eq}}$, with characteristic time $\tau_{j 2}$ [see Eq. (1)]. Note that for the thermal binary-mixture model of Ref. [18] both species $\mathrm{A}$ and $\mathrm{B}$ have equal characteristic times $\tau_{A 1}=\tau_{B 1}$ and $\tau_{A 2}=\tau_{B 2}$, which results in common transport properties. The introduction of variable characteristic times $\tau_{j 1}$ and $\tau_{j 2}$ allows every component $j$ to have its own transport properties.

The relevant moments of each species, which are necessary in the following analysis, are the density, momentum, energy, pressure tensor, third-order moment, third-order contracted moment, and fourth-order contracted moment,

$$
\begin{aligned}
\rho_{j} & =\sum_{i=0}^{8} f_{j i}, \quad J_{j \alpha}=\sum_{i=0}^{8} f_{j i} c_{j i \alpha}, \quad E_{j}=\sum_{i=0}^{8} f_{j i} c_{j i}^{2}, \\
P_{j \alpha \beta} & =\sum_{i=0}^{8} f_{j i} c_{j i \alpha} c_{j i \beta}, \quad Q_{j \alpha \beta \gamma}=\sum_{i=0}^{8} f_{j i} c_{j i \alpha} c_{j i \beta} c_{j i \gamma}, \\
q_{j \alpha} & =\sum_{i=0}^{8} f_{j i} c_{j i \alpha} c_{j i}^{2}, \quad R_{j \alpha \beta}=\sum_{i=0}^{8} f_{j i} c_{j i \alpha} c_{j i \beta} c_{j i}^{2} .
\end{aligned}
$$

The equilibrium distribution function $f_{j i}^{\mathrm{eq}}$ is found by minimizing the $\mathrm{H}$ function under specific constraints given as [18,32-34]

$$
\begin{aligned}
f_{j i}^{\mathrm{eq}}\left(\rho_{j}, u, T\right)= & \rho_{j} \prod_{\alpha=x, y} \frac{\left(1-2 c_{0 i \alpha}^{2}\right)}{(2)^{C_{0 i \alpha}^{2}}}\left[\left(c_{0 i \alpha}^{2}-1\right)\right. \\
& \left.+\sqrt{M_{j}} c_{0 i \alpha} u_{\alpha}+M_{j} u_{\alpha}^{2}+T\right],
\end{aligned}
$$

where $c_{0 i}$ is defined via Eq. (2) by setting $c_{0}=1 ; u_{\alpha}$ is the mixture velocity, which is related to the mixture momentum via $u_{\alpha}=J_{\alpha} / \rho$, with $\rho$ the mixture density; $C$ is the concentration of the mixture, defined as $C=\sum_{j}^{N} C_{j}=\sum_{j}^{N} \rho_{j} / M_{j}$; and $T$ is the temperature of the mixture and is related with the total energy of the mixture $E$. The total mixture momentum and energy can be calculated by

$$
\begin{aligned}
& J_{\alpha}=\sum_{j}^{N} J_{j \alpha}=\sum_{j}^{N} \sum_{i=0}^{8} f_{j i} c_{j i \alpha}, \\
& E=2 C T+\frac{J^{2}}{\rho}=\sum_{j}^{N} E_{j}=\sum_{j}^{N} \sum_{i=0}^{8} f_{j i} c_{j i}^{2} .
\end{aligned}
$$

The moments of the equilibrium distribution function are in agreement to those predicted by the kinetic theory of gases [Maxwell-Boltzmann form (MB)] [35] up to second order. Deviations appear in the higher order moments due to the simplicity of the employed lattice in the discretization and are indicated as $Q_{j \alpha \beta \gamma}^{\prime}, q_{j \alpha}^{\prime}$ and $R_{j \alpha \beta}^{\prime}$, respectively (see Appendix A). These deviations will influence the macroscopic limit of the kinetic model. Nevertheless, proper counterterms $\psi_{j i}$ and $\phi_{j i}$ can be introduced at the level of the lattice BGK equation, in a way presented in Refs. [34,36,37] and similar to the thermal binarymixing model [18]. Their purpose is to neutralize the inherent deviation in the macroscopic limits and to ensure the recovery of the correct thermohydrodynamics. The term $\psi_{j i}$ is used to reproduce the correct momentum equation, while $\phi_{j i}$ is used for reproducing the energy equation. The exact forms of these two terms are identified through the Chapman-Enskog analysis of the kinetic equation, and are given in the next section.

The auxiliary state population $f_{j i}^{*}$ has a similar form as $f_{j i}^{\mathrm{eq}}$ but with the mixture velocity replaced by the individual species velocity and reads as

$$
\begin{aligned}
f_{j i}^{*}\left(\rho_{j}, u_{j}, T\right)= & \rho_{j} \prod_{\alpha=x, y} \frac{\left(1-2 c_{0 i \alpha}^{2}\right)}{(2)^{C_{0 i \alpha}^{2}}}\left[\left(c_{0 i \alpha}^{2}-1\right)\right. \\
& \left.+\sqrt{M_{j}} c_{0 i \alpha} u_{j \alpha}+M_{j} u_{j \alpha}^{2}+T\right],
\end{aligned}
$$

where $u_{j \alpha}$ is the velocity of species $j$ defined as $u_{j \alpha}=J_{j \alpha} / \rho_{j}$.

The final discretized kinetic expression of Eq. (1) can be obtained by applying the trapezoidal rule and is reformulated with the help of the transformation population $g_{j i}$ (transforms the scheme to an explicit one), which gives

$$
\begin{aligned}
g_{j i}(t+\delta t)= & g_{j i}(t)-\frac{2 \delta t}{\delta t+2 \tau_{j 1}}\left[g_{j i}(t)-f_{j i}^{*}(t)\right] \\
& -\frac{2 \delta t}{\delta t+2 \tau_{j 1}} \frac{\tau_{j 1}}{\tau_{j 2}}\left[f_{j i}^{*}(t)-f_{j i}^{\mathrm{eq}}(t)\right] \\
& +\frac{2 \tau_{j 1} \delta t}{\delta t+2 \tau_{j 1}}\left[\psi_{j i}(t)+\phi_{j i}(t)\right],
\end{aligned}
$$

where for the usual scheme, $\delta t=1$. The transformation population, $g_{j i}$, is defined as

$$
\begin{aligned}
g_{j i}= & f_{j i}+\frac{\delta t}{2 \tau_{j 1}}\left[f_{j i}-f_{j i}^{*}\right]+\frac{\delta t}{2 \tau_{j 2}}\left[f_{j i}^{*}-f_{j i}^{\mathrm{eq}}\right] \\
& -\frac{\delta t}{2}\left[\psi_{j i}+\phi_{j i}\right] .
\end{aligned}
$$

The local density, momentum, and temperature of population $f_{j i}$ are therefore related with the populations $g_{j i}$, respectively 
as follows:

$$
\begin{aligned}
\rho_{j}(f)= & \rho_{j}(g), \\
J_{j \alpha}(f)= & \frac{J_{j \alpha}(g)+\frac{\delta t}{2 \tau_{j 2}} J_{j \alpha}^{\mathrm{eq}}(f)+\frac{\delta t}{2} \sum_{i=0}^{8} c_{j i \alpha} \psi_{j i}}{\left(1+\frac{\delta t}{2 \tau_{j 2}}\right)}, \\
T(f)= & \frac{E(f)-\frac{J(f)^{2}}{\rho(f)}}{2 C}=\frac{E(g)-\frac{J(g)^{2}}{\rho(g)}}{2 C} \\
& +\frac{\delta t}{4 C} \sum_{i=0, j}^{i=8, j=N} c^{2} \phi_{j i} .
\end{aligned}
$$

\section{MACROSCOPIC LIMIT}

The macroscopic limit of the proposed kinetic equation Eq. (1) is studied via Chapman-Enskog expansion analysis. By taking the moments of the kinetic equation Eq. (1) and using the definition of the moments of the distribution function $f_{j i}$ as shown in Eq. (3), the moment transport equations are obtained for each species as well as for the mixture. The density $\rho_{j}$, momentum $J_{j \alpha}$, and energy $E_{j}$ transport equations of each species $j$ are the following:

Individual density:

$$
\partial_{t} \rho_{j}+\partial_{\alpha} J_{j \alpha}=0 .
$$

Individual momentum:

$$
\partial_{t} J_{j \alpha}+\partial_{\beta} P_{j \alpha \beta}=-\frac{V_{j \alpha}}{\tau_{j 2}} .
$$

Individual pressure tensor:

$$
\begin{aligned}
\partial_{t} & P_{j \alpha \beta}+\partial_{\gamma} Q_{j \alpha \beta \gamma} \\
& =\frac{1}{\tau_{j 1}}\left(P_{j \alpha \beta}^{*}-P_{j \alpha \beta}\right)+\frac{1}{\tau_{j 2}}\left(P_{j \alpha \beta}^{\mathrm{eq}}-P_{j \alpha \beta}^{*}\right) .
\end{aligned}
$$

Individual third-order contracted moment:

$$
\partial_{t} q_{j \alpha}+\partial_{\beta} R_{j \alpha \beta}=\frac{1}{\tau_{j 1}}\left[q_{j \alpha}^{*}-q_{j \alpha}\right]+\frac{1}{\tau_{j 2}}\left[q_{j \alpha}^{\mathrm{eq}}-q_{j \alpha}^{*}\right] .
$$

In the following analysis, the moment transport equations are expanded to different orders. On the zeroth order, transport equations of the nonconserved moments of each species $j$ are identified as

$$
\begin{gathered}
\partial_{t}^{(0)} J_{j \alpha}+\partial_{\beta} P_{j \alpha \beta}^{\mathrm{eq}}=-\frac{V_{j \alpha}^{(1)}}{\tau_{j 2}}, \\
\partial_{t}^{(0)} P_{j \alpha \beta}^{\mathrm{eq}}+\partial_{\gamma} Q_{j \alpha \beta \gamma}^{\mathrm{eq}}=-\frac{1}{\tau_{j 1}} P_{j \alpha \beta}^{(1)}+\left(\frac{1}{\tau_{j 1}}-\frac{1}{\tau_{j 2}}\right) \\
\times\left[\left(u_{\alpha} V_{j \beta}^{(1)}+u_{\beta} V_{j \alpha}^{(1)}\right)\right], \\
\partial_{t}^{(0)} q_{j \alpha}^{\mathrm{eq}}+\partial_{\beta} R_{j \alpha \beta}^{\mathrm{eq}} \\
=-\frac{1}{\tau_{j 1}} q_{j \alpha}^{(1)}+\left(\frac{1}{\tau_{j 1}}-\frac{1}{\tau_{j 2}}\right) \frac{4}{M_{j}} T V_{j \alpha}^{(1)} \\
+\left(\frac{1}{\tau_{j 1}}-\frac{1}{\tau_{j 2}}\right)\left(J_{j \alpha} u_{j}^{2}-Y_{j} J_{\alpha} u^{2}+q_{j \alpha}^{\prime *}-q_{j \alpha}^{\prime e q}\right) .
\end{gathered}
$$

Note that in the case of the binary mixture of Ref. [18], when summing up the equivalent Eq. (15) over species $j$, the second terms on the right-hand-side (RHS) sum up to zero. For the present bare model, however, they do not sum up to zero, due to different relaxation times for each species, and they show up as deviation terms that affect the macroscopic limit. Similarly, the third term on the RHS in Eq. (16) results in an undesired deviation term. The influence of both aforementioned terms on the macroscopic momentum and energy equation will be identified through the Chapman-Enskog analysis and will be finally neutralized.

\section{A. Mass diffusivity}

In order to derive the macroscopic multicomponent diffusion equation and identify the relation between the binary diffusivities, $D_{j k}$, and the relaxation time, $\tau_{j 2}$, for each species, Eq. (14) is further analyzed. Taking the zeroth-order transport equations of different moments into account [18], substituting with Eq. (A1), and using the definition of diffusion flux $V_{j \alpha}=J_{j \alpha}-\left(\rho_{j} / \rho\right) J_{\alpha}$, one can get the first-order component of the diffusion flux $V_{j \alpha}^{(1)}$ using Eq. (14):

$$
V_{j \alpha}^{(1)}=\tau_{j 2}\left[Y_{j} \partial_{\alpha} p-\partial_{\alpha} p_{j}\right]
$$

where $p_{j}=C_{j} T$ is the pressure of species $j$. Since the diffusion flux at the equilibrium state is zero, $V_{j \alpha}^{\mathrm{eq}}=0$, Eq. (17) can be rewritten in the following form:

$$
\partial_{\alpha} X_{j}=-\frac{V_{j \alpha}}{\tau_{j 2} p}+\frac{Y_{j}-X_{j}}{p} \partial_{\alpha} p .
$$

Comparing this equation with the multicomponent diffusion equation (in the absence of body forces and neglecting the Soret effect),

$$
\partial_{\alpha} X_{j}=\sum_{k=1}^{N} \frac{X_{j} X_{k}}{D_{j k}}\left(u_{k \alpha}-u_{j \alpha}\right)+\left(Y_{j}-X_{j}\right) \frac{\partial_{\alpha} p}{p},
$$

leads to the relation between binary diffusivity $D_{j k}$ and relaxation time $\tau_{j 2}$. Similar to Ref. [38], the relation is

$$
\tau_{j 2}=\frac{\rho_{j}}{P_{j}} D_{j m}
$$

where $D_{j m}$ is the mixture-averaged diffusion coefficient of species $j[38,39]$,

$$
D_{j m}=\frac{1-Y_{j}}{\sum_{k \neq j}^{N} X_{k} / D_{j k}},
$$

which is based on the mixture-averaged diffusion approximation. This approximation is widely used in many thermofluidic applications such as combustion processes [40,41]. In some isothermal multicomponent LB models this approximation is not invoked [42-44]. A deviation is introduced to the mixture momentum equation, whose RHS becomes nonzero:

$$
\partial_{t} J_{\alpha}+\partial_{\beta} P_{\alpha \beta}=-\sum_{j}^{N} \frac{V_{j \alpha}}{\tau_{j 2}} \neq 0 .
$$

In order to restore conservation of the local mixture momentum, a correction velocity $U_{c \alpha}$ is used [38],

$$
J_{j \alpha}=\tilde{J}_{j \alpha}+\rho_{j} U_{c \alpha}
$$

where $\tilde{J}_{j \alpha}$ is the momentum of species $j$ before the correction velocity implementation on the momentum conservation, and 
$J_{j \alpha}$ is the desired corrected momentum of species $j$. The correction velocity $U_{c \alpha}$ has the following property:

$$
\sum_{j}^{N} \frac{\rho_{j} U_{c \alpha}}{\tau_{j 2}}=-\sum_{j}^{N} \frac{\frac{\rho_{j}}{\rho} J_{\alpha}-\tilde{J}_{j \alpha}}{\tau_{j 2}} .
$$

The correction velocity is applied to the momentum equation by adding the forcing term $\psi_{j i}^{(\mathrm{I})}$ into the kinetic equation (1),

$$
\psi_{j i}^{(\mathrm{I})}=\Psi_{j i \alpha} \frac{\rho_{j} U_{c \alpha}}{\tau_{j 2}},
$$

where the coefficients $\Psi_{j i \alpha}(\alpha=x, y)$ are defined as

$$
\begin{aligned}
\Psi_{j i x} & =\frac{1}{4 c_{j}}\{0,4,0,-4,0,-1,1,1,-1\}, \\
\Psi_{j i y} & =\frac{1}{4 c_{j}}\{0,0,4,0,-4,-1,-1,1,1\} .
\end{aligned}
$$

\section{B. Viscosity}

In order to derive the macroscopic momentum equation and identify the relation between the dynamic viscosity $\mu_{j}$ and the relaxation parameter $\tau_{j 1}$ for each species, Eq. (15) is the starting point. The nonequilibrium pressure tensor $P_{\alpha \beta}^{\mathrm{eq}}$ is obtained as

$$
\begin{aligned}
P_{\alpha \beta}^{(1)}= & -\sum_{j}^{N}\left(\tau_{j 1} C_{j}\right) T \partial_{\gamma}\left(\frac{J_{\alpha}}{\rho} \delta_{\beta \gamma}+\frac{J_{\beta}}{\rho} \delta_{\alpha \gamma}-\frac{J_{\gamma}}{\rho} \delta_{\alpha \beta}\right) \\
& +P_{\alpha \beta}^{\prime \prime} .
\end{aligned}
$$

In the derivation, special care is taken for the second term on the RHS of Eq. (15). If the relaxation times $\tau_{j 1}$ have the same value for each species (e.g., the binary-mixture model), this term will sum up to zero for the total mixture and will not introduce any deviation at the macroscopic momentum equation. On the contrary, if $\tau_{j 1}$ is different for each species (the case of a multicomponent mixture), the summation of this term for the mixture does not add up to zero, thus introducing a deviation at the macroscopic momentum equation limit. Finally, the total deviation of the nonequilibrium pressure tensor of the total mixture, $P_{\alpha \beta}^{\prime \prime}$, is obtained as

$$
\begin{aligned}
P_{\alpha \beta}^{\prime \prime}= & -\sum_{j}^{N} \tau_{j 1} \partial_{\gamma} Q_{j \alpha \beta \gamma}^{\prime}+\sum_{j}^{N} \frac{\tau_{j 1} X_{j}}{2} \partial_{\gamma} q_{\gamma}^{\prime} \delta_{\alpha \beta} \\
& +\sum_{j}^{N}\left(u_{\alpha} V_{j \beta}^{(1)}+u_{\beta} V_{j \alpha}^{(1)}\right) .
\end{aligned}
$$

The final macroscopic momentum equation of the bare kinetic model (without considering the correction terms) is

$$
\begin{aligned}
& \partial_{t} J_{\alpha}+\partial_{\alpha} p+\partial_{\beta}\left[\frac{J_{\alpha} J_{\beta}}{\rho}-\sum_{j}^{N}\left(\tau_{j 1} C_{j}\right) T \partial_{\gamma}\right. \\
& \left.\quad \times\left(\frac{J_{\alpha}}{\rho} \delta_{\beta \gamma}+\frac{J_{\beta}}{\rho} \delta_{\alpha \gamma}-\frac{J_{\gamma}}{\rho} \delta_{\alpha \beta}\right)\right]+\partial_{\beta} P_{\alpha \beta}^{\prime \prime}=0 .
\end{aligned}
$$

The specific form of the deviation term $\partial_{\beta} P_{\alpha \beta}^{\prime \prime}$ is given in Appendix B 1 and is neutralized by using the counterterm $\psi_{j i \alpha}^{(\text {II) }}$ :

$$
\psi_{j i}^{(\mathrm{II})}=\Psi_{j i \alpha} \partial_{\beta} P_{\alpha \beta}^{\prime \prime} .
$$

Therefore, the total correction that needs to be applied to the kinetic equation for the recovery of the correct momentum equation is

$$
\psi_{j i}=\psi_{j i}^{(\mathrm{I})}+\psi_{j i}^{(\mathrm{II})} .
$$

The dynamic viscosity of the mixture can be identified as

$$
\mu=\sum_{j}^{N}\left(\tau_{j 1} C_{j}\right) T \text {. }
$$

For the exact relation between dynamic viscosity $\mu_{j}$ and the relaxation time $\tau_{j}$ for each species, the Wilke formula [40,45] is used. It defines the viscosity of a multicomponent mixture in terms of concentration, viscosity, and molecular weight of each component,

$$
\mu=\sum_{j}^{N} \frac{X_{j} \mu_{j}}{\sum_{k}^{N} X_{k} \varphi_{j k}}
$$

where $\varphi_{j k}$ is

$$
\varphi_{j k}=\frac{1}{\sqrt{8}}\left(1+\frac{M_{j}}{M_{k}}\right)^{-1 / 2}\left[1+\left(\frac{\mu_{j}}{\mu_{k}}\right)^{1 / 2}\left(\frac{M_{k}}{M_{j}}\right)^{1 / 4}\right]^{2} .
$$

By comparing Eq. (32) and Wilke formula (33), the relation between $\mu_{j}$ and $\tau_{j 1}$ is

$$
\tau_{j 1}=\frac{\mu_{j}}{p \sum_{k}^{N} X_{k} \varphi_{j k}} .
$$

\section{Heat conductivity of the bare model}

In the analysis of the energy equation, Eq. (16) is used to calculate the heat conductivity. The nonequilibrium contracted third-order moment $q_{\alpha}^{(\text {neq) }}$ is shown to be

$$
\begin{aligned}
q_{\alpha}^{(\text {neq })}= & -\left[\begin{array}{l}
4 p \sum_{j}^{N} \frac{X_{j} \tau_{j 1}}{M_{j}} \partial_{\alpha} T+4 T \sum_{j}^{N} \frac{V_{j \alpha}}{M_{j}} \\
-2 p \sum_{j}^{N} X_{j} \tau_{j 1} u_{\beta}\left(\partial_{\alpha} u_{\beta}+\partial_{\beta} u_{\alpha}-\partial_{\gamma} u_{\gamma} \delta_{\alpha \beta}\right)
\end{array}\right] \\
& +q_{\alpha}^{\prime \prime},
\end{aligned}
$$

where $q_{\alpha}^{\prime \prime}$ is the deviation of the nonequilibrium third-order contracted moment $q_{\alpha}^{(\text {neq) }}$ from the desired MB form. In the bare thermal multicomponent model (before the introduction of the energy correction terms), the recovered macroscopic energy equation is obtained and given in terms of temperature evolution as follows:

$$
\begin{aligned}
\partial_{t} T=- & T \partial_{\alpha} u_{\alpha}-u_{\alpha} \partial_{\alpha} T+\frac{1}{C} \partial_{\alpha}\left(2 p \sum_{j}^{N} \frac{X_{j} \tau_{j 1}}{M_{j}} \partial_{\alpha} T\right) \\
& -\frac{1}{C} \partial_{\alpha} \sum_{j}\left(2 T C_{j} U_{j \alpha}\right)+\frac{1}{C} \sum_{j}^{N}\left(X_{j} \tau_{j 1}\right) p \partial_{\alpha} u_{\beta} \\
& \times\left(\partial_{\alpha} u_{\beta}+\partial_{\beta} u_{\alpha}-\partial_{\gamma} u_{\gamma} \delta_{\beta \alpha}\right)-\frac{1}{2 C}\left(\partial_{\alpha} q_{\alpha}^{\prime}+\partial_{\alpha} q_{\alpha}^{\prime \prime}\right),
\end{aligned}
$$

where $U_{j \alpha}=V_{j \alpha} / \rho_{j}$ is the diffusion velocity. The heat conductivity $\kappa$ of the gas mixture for the bare LB model is identified as

$$
\kappa=2 p \sum_{j}^{N} \frac{X_{j} \tau_{j 1}}{M_{j}}
$$


In Eq. (37), the last term on the RHS is the deviation of the bare LB model from the desired macroscopic limit. It is corrected by introducing a counter term $\phi_{j i}^{(\mathrm{I})}$ at the kinetic equation through a forcing scheme, defined as

$$
\phi_{j i}^{(\mathrm{I})}=\Phi_{j i} \partial_{\alpha}\left(q_{\alpha}^{\prime}+q_{\alpha}^{\prime \prime}\right),
$$

where the coefficient $\Phi_{j i}$ is

$$
\Phi_{j i}=\frac{1}{8 c_{j}^{2}}\{-12,4,4,4,4,-1,-1,-1,-1\} .
$$

$q_{\alpha}^{\prime}$ is given in Appendix $\mathrm{A}$, and $q_{\alpha}^{\prime \prime}$ is identified through Chapman-Enskog analysis in Appendix B 2. Since the exact form of this term is known, it is neutralized by using the forcing scheme for $\phi_{j i}$ similar to Ref. [18,34].

\section{Variable Prandtl number and heat conductivity}

In the previous section, the heat conductivity of the mixture stemming from the bare LB model leads to a fixed Prandtl number (Pr) value. The $\operatorname{Pr}$ and heat conductivity can be adjusted by adding $q_{j \alpha}^{\mathrm{Pr}}$ in the correction terms. The implementation is done through the forcing term $\phi_{j i}^{(\mathrm{II})}=\partial_{\alpha} q_{j \alpha}^{\mathrm{Pr}}$, which affects the energy equation. The $q_{j \alpha}^{\mathrm{Pr}}$ for each species reads

$$
q_{j \alpha}^{\operatorname{Pr}}=\left(\frac{4}{\operatorname{Pr} \bar{M}}-\frac{4}{M_{j}}\right) X_{j} \tau_{j 1} p \partial_{\alpha} T,
$$

and therefore the heat conductivity of the mixture becomes

$$
\kappa=\frac{2 p}{\operatorname{Pr} \bar{M}} \sum_{j}^{N} \tau_{j 1} X_{j} .
$$

The Prandtl number can also be adjusted for each species. If the Pr number of species $j$ is known and denoted as $\operatorname{Pr}_{j}$, the responsible term $q_{j \alpha}^{\mathrm{Pr}}$ for species $j$ takes the following form:

$$
q_{j \alpha}^{\operatorname{Pr}_{j}}=\frac{4 \tau_{j 1} \sum_{k}^{N}\left(X_{k} \phi_{j k}\right)}{\operatorname{Pr}_{j} M_{j}} p \partial_{\alpha} T-\frac{4 X_{j} \tau_{j 1}}{M_{j}} C T \partial_{\alpha} T,
$$

which leads to the corresponding heat conductivity of each species as

$$
\kappa_{j}=\frac{2 \tau_{j 1} p \sum_{k}^{N} X_{k} \phi_{j k}}{\operatorname{Pr}_{j} M_{j}} .
$$

In general, the mixture-averaged thermal conductivity calculated by CHEMKIN package $[39,46]$ can be applied. It is evaluated through the Mathur formula [39,47]:

$$
\kappa=\frac{1}{2}\left(\sum_{j=1}^{N} X_{j} \kappa_{j}+\frac{1}{\sum_{j=1}^{N} X_{j} / \kappa_{j}}\right) .
$$

Equations (41) and Eq. (42) are used to conveniently control the Prandtl number and heat conductivity of the mixture.

Finally, the term that can guarantee the recovery of the correct macroscopic energy equation and a tunable Prandtl number is

$$
\phi_{j i}=\phi_{j i}^{(\mathrm{I})}+\phi_{j i}^{(\mathrm{II})}
$$

\section{SPEED OF SOUND}

In this section the ability of the proposed model to describe the speed of sound in a multicomponent mixture (thermal) is examined. LB multicomponent models can describe the propagation of sound waves only at an athermal environment. To the best of our knowledge the only single-component thermal LB model on the D2Q9 which is able to capture accurately the speed of sound in a thermal environment is the model of Ref. [34]. The model presented in this paper extends this description to multicomponent flows on the D2Q9. To study this behavior we measure the propagation of small pressure waves in gaseous mixtures at different temperatures and compositions.

For the simulation setup, a mixture composed of different species is considered. The computational domain is discretized by $N_{x} \times N_{y}=3000 \times 5$ grid points along the horizontal $x$ and vertical $y$ direction, respectively. At the left and right boundaries of the domain, zero-Neumann BCs are applied by copying the populations from the neighboring nodes in the inner domain. The top and bottom boundaries are treated with periodic boundary conditions. The simulation is repeated for different temperatures and compositions of species in four sets, S-I to S-IV, as is described in Tables I and II. In each simulation, the initial temperature and species concentration distributions are uniform through the domain. In the first two cases a binary mixture composed of species $A$ and $B$ is considered. Cases $\mathrm{S}$-III and S-IV refer to a mixture composed of four species. For all cases the molecular mass of the species can be freely defined over a large range.

Figure 1 shows the speed of sound at different temperatures for the binary gaseous mixture under the condition of case S-I. The binary mixture is composed of $50 \%$ species $A$ and $50 \%$ species $B$, with a mean molar mass $\bar{M}=2.5$ (in LB units). The LB simulation results (symbols in Fig. 1) are compared with the theoretical value (solid line in Fig. 1) given by

$$
c_{s}=\sqrt{\frac{\gamma R T}{\bar{M}}},
$$

where $\gamma=c_{P} / c_{V}$ is the specific heat ratio with $c_{P}$ being the specific heat at constant pressure and with $c_{V}$ being the specific

TABLE I. Conditions of cases S-I and S-II for the sound speed of binary gaseous mixtures.

\begin{tabular}{lccccc}
\hline \hline Simulation & \multicolumn{3}{c}{ Gaseous mixture } & Temperature \\
\hline Cases & Species & $X_{j}$ & $M_{j}$ & $\bar{M}^{\mathrm{a}}$ & T \\
& & $(\%)$ & $(\mathrm{LB}$ units $)$ & $(\mathrm{LB}$ units $)$ & $(\mathrm{LB}$ units $)$ \\
S-I & $A$ & 50 & 1 & 2.5 & {$[0.001, \ldots, 0.999]$} \\
& $B$ & 50 & 4 & & 0.5 \\
S-II & $A$ & 60 & 1 & {$[1, \ldots, 400.6]$} & \\
& $B$ & 40 & {$[1, \ldots, 1000]$} & & \\
\hline \hline
\end{tabular}

${ }^{\mathrm{a}} \bar{M}$ is the mean molar mass of the mixture. 
TABLE II. Conditions of cases S-III and S-IV for the sound speed of four-component gaseous mixtures.

\begin{tabular}{|c|c|c|c|c|c|}
\hline \multirow{2}{*}{$\frac{\text { Simulation }}{\text { Cases }}$} & \multicolumn{4}{|c|}{ Gaseous mixture } & \multirow{2}{*}{$\begin{array}{c}\text { Temperature } \\
\mathrm{T} \\
(\mathrm{K})\end{array}$} \\
\hline & Species & $\begin{array}{l}X_{j} \\
(\%)\end{array}$ & $\begin{array}{c}M_{j} \\
(\mathrm{~g} / \mathrm{mol})\end{array}$ & $\begin{array}{c}\bar{M}^{\mathrm{a}} \\
(\mathrm{g} / \mathrm{mol})\end{array}$ & \\
\hline \multirow[t]{4}{*}{ S-III } & $\mathrm{H}_{2}$ & {$[5, \ldots, 97]$} & 2 & {$[2.74, \ldots, 41.37]$} & 800 \\
\hline & $\mathrm{CH}_{4}$ & {$[1, \ldots, 35]$} & 16 & & \\
\hline & $\mathrm{CO}_{2}$ & {$[1, \ldots, 93]$} & 44 & & \\
\hline & $\mathrm{H}_{2} \mathrm{O}$ & {$[1, \ldots, 15]$} & 18 & & \\
\hline \multirow[t]{4}{*}{ S-IV } & $\mathrm{N}_{2}$ & 20 & 28 & 32.6 & {$[400, \ldots, 3000]$} \\
\hline & $\mathrm{CH}_{4}$ & 20 & 16 & & \\
\hline & $\mathrm{CO}_{2}$ & 50 & 44 & & \\
\hline & $\mathrm{H}_{2} \mathrm{O}$ & 10 & 18 & & \\
\hline
\end{tabular}

${ }^{\mathrm{a}} \bar{M}$ is the mean molar mass of the mixture.

heat at constant volume [in the model, $c_{P}=(D+2) / 2$ and $c_{V}=D / 2$, giving for a two-dimensional $(D=2)$ case $\gamma=2$ and for a three-dimensional case $(D=3) \gamma=5 / 3$ ], and $R$ is the universal gas constant (in the model, $R=1$ in LB units). We note at this point that in LB units the temperature can vary between 0 and 1 , and that all isothermal models on the D2Q9 lattice operate by construction at the frozen temperature $T=1 / 3$. The flexibility of the model to operate at arbitrary temperatures stems from the thermal construction in conjunction with the implementation of appropriate correction terms on the level of the energy equation.

The results for case S-II are presented in Fig. 2. The sound speed is plotted for binary mixtures of different compositions and at temperature $T=0.5$ (in LB units). The composition is varied by changing the molar mass of species $B$ from 1 to 1000 with a constant mole fraction $40 \%$, while species $A$ has $M_{A}=1$ (in LB units) and mole fraction $60 \%$. Therefore, the molecular mass ratio of two species ranges from 1 to $1 / 1000$, and accordingly the mean molecular weight $\bar{M}$ spans the range 1 to 400.6 (in LB units). For both S-I and S-II cases, the simulation results of the current model (symbols in Figs. 1 and 2) are in excellent agreement with the theoretically predicted values.

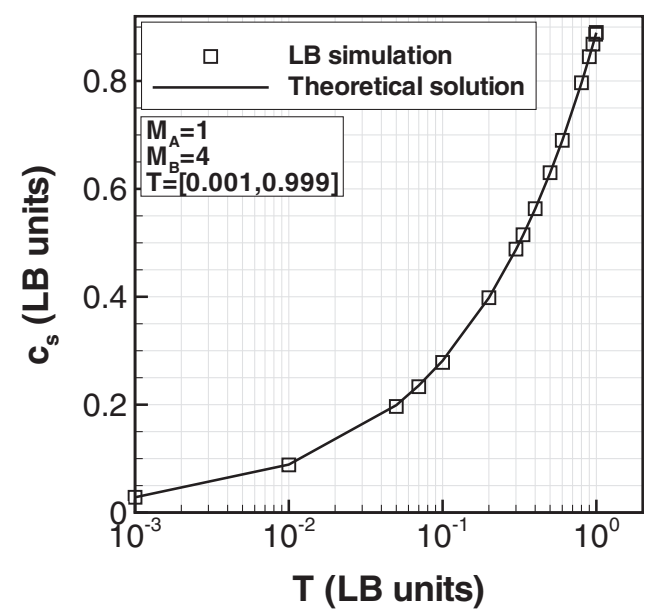

FIG. 1. Case S-I: Speed of sound computed for a binary gaseous mixture at different temperatures. Symbols: LB simulation, solid line: theoretical solution.
Figure 3 shows simulation results for case S-III. The mixture is composed of four species: $\mathrm{H}_{2}, \mathrm{CH}_{4}, \mathrm{CO}_{2}$, and $\mathrm{H}_{2} \mathrm{O}$. The species mole fractions are adjusted arbitrarily, and their values are shown in Fig. 3(a), leading to corresponding values of mean molar mass ranging from $2.74 \mathrm{~g} / \mathrm{mol}$ to $41.37 \mathrm{~g} / \mathrm{mol}$ (in physical units). The temperature is fixed at $800 \mathrm{~K}$, with reference temperature $T_{\text {ref,phy }}=1500 \mathrm{~K}$, which corresponds to $T_{\text {ref,LB }}=1 / 3$ in LB units in the current formulation. (For unit transformation, refer to Appendix C.)

Figure 4 shows the simulation result for case S-IV. The mixture is composed of a different set of four species with a fixed mole fraction composition: $\mathrm{N}_{2}(20 \%), \mathrm{CH}_{4}(20 \%), \mathrm{CO}_{2}$ (50\%), and $\mathrm{H}_{2} \mathrm{O}(10 \%)$. The mean molar mass is $32.6 \mathrm{~g} / \mathrm{mol}$. The speed of sound is calculated at temperatures from 400 to $3000 \mathrm{~K}$. (The reference temperature is the same as in case S-III.) The simulation results of cases S-III and S-IV verify that the speed of sound is described correctly in multispecies mixtures for a large range of temperatures, compositions and species molecular masses.

Therefore, the proposed thermal multicomponent model can be potentially used to simulate propagation of acoustic waves. This can be quite useful for the study of

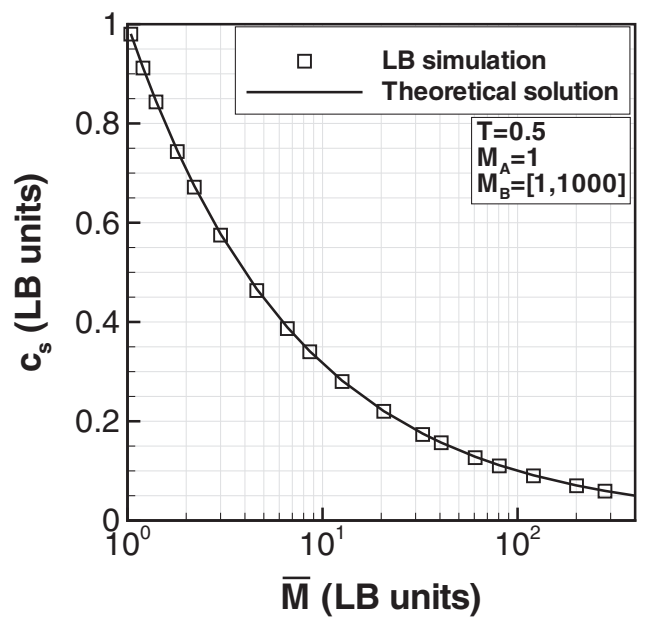

FIG. 2. Case S-II: Speed of sound computed for binary gaseous mixtures with different compositions at the same temperature. Symbols: LB simulation, solid line: theoretical solution. 

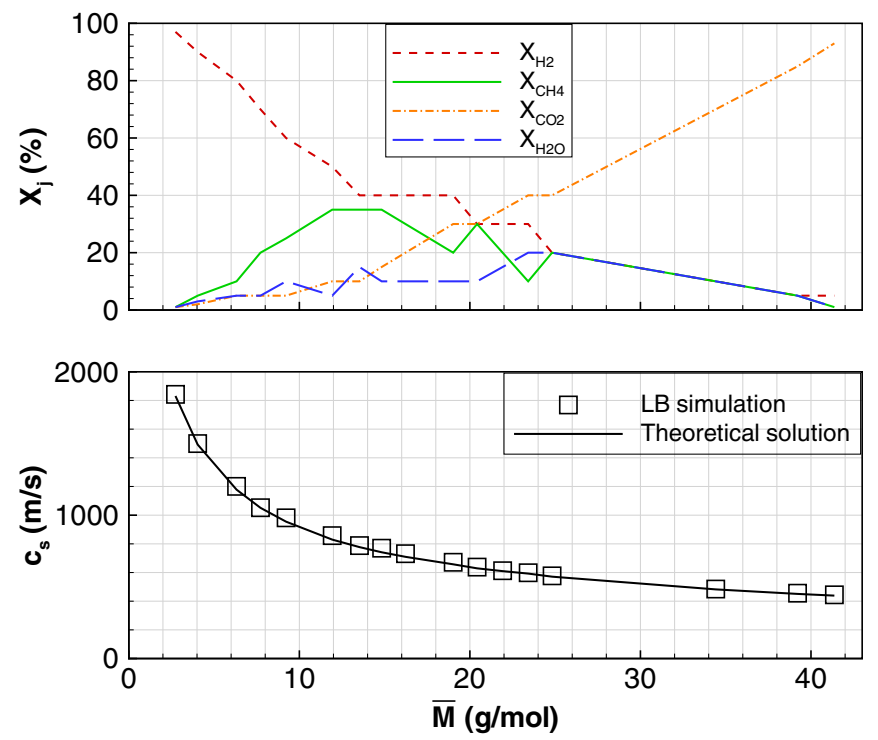

FIG. 3. (Color online) Case S-III: Speed of sound computed for gaseous mixtures composed of four species with different composition at $T=800 \mathrm{~K}$. Top figure: Mole fractions of each species, dashed line represents $\mathrm{H}_{2}$, solid line represents $\mathrm{CH}_{4}$, dash-dotted line represents $\mathrm{CO}_{2}$, long dashed line represents $\mathrm{H}_{2} \mathrm{O}$. Bottom figure: Symbols represent LB simulation, solid line represents theoretical solution.

thermoacoustics in combustion [48]. It is worth commenting that the benchmarks of this section have also demonstrated the good numerical stability of the model.

\section{BOUNDARY SCHEME FOR THERMAL CATALYTIC SURFACE REACTION}

The proposed model recovers the correct macroscopic limits for describing thermal multicomponent flows; moreover, the physical properties of the species can be freely adjusted. Therefore, this model can be applied in a variety of relatively complex flow problems such as the catalytic reactive flows with

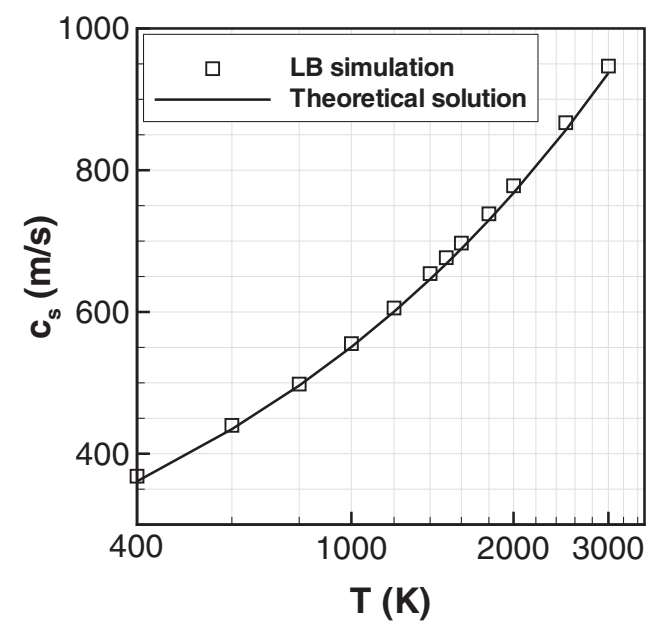

FIG. 4. Case S-IV: Speed of sound computed for gaseous mixtures composed of four species at different temperatures. Symbols: LB simulation, solid line: theoretical solution.

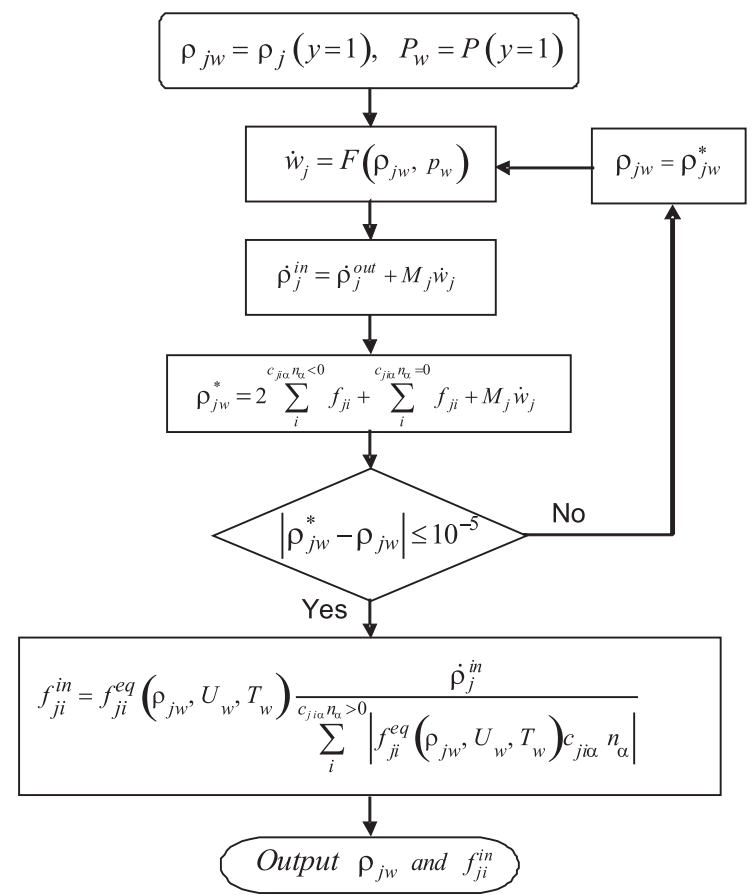

FIG. 5. Schematic for calculating the unknown density $\rho_{j w}$ and incoming distribution function $f_{j i}$ for species $j$ at the catalytic wall.

large temperature variations. In order to accurately describe the effect of catalytic reactions and at the same time to vary the wall temperature, the catalytic diffusive boundary condition described in Refs. [21,25,49] needs to be extended.

In this section the diffusive boundary condition [25,49], which has been modified to include surface chemistry under isothermal conditions in [21], is further extended to describe thermal wall boundaries. In the diffusive boundary condition, the populations are redistributed after they reach the boundary in a way consistent with the mass balance and normal flux conditions. For the evaluation of the unknown precollision incoming populations $f_{j i}^{\text {in }}$ on the catalytic wall boundary, the first constraint is established by utilizing the property of mass conservation at the wall. During the chemical reaction on the surface (no deposition or etching effect is considered on the surface, such that the Stefan velocity is zero), mass is balanced among the incoming mass fluxes $\dot{\rho}_{j}^{\text {in }}$, outgoing fluxes $\dot{\rho}_{j}^{\text {out }}$, and the production rate $\dot{\omega}_{j}$ of species $j$ :

$$
\dot{\rho}_{j}^{\text {in }}=\dot{\rho}_{j}^{\text {out }}+M_{j} \dot{\omega}_{j},
$$

where $\dot{\omega}_{j}$ is a function of $C_{j w}=\rho_{j w} / M_{j}$, with $C_{j w}$ being the local concentration of species $j$ at the gas-wall interface, and

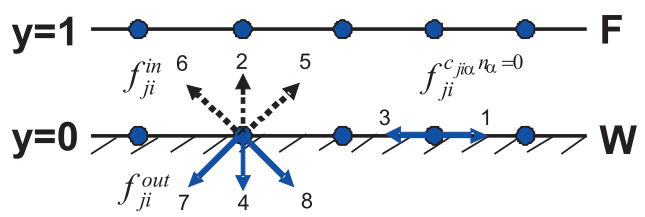

FIG. 6. (Color online) Boundary condition on a catalytic wall. "W" denotes wall boundary at $y=0$, "F" denotes the first row of fluid nodes at $y=1$, and bold lattice nodes indicate that the collision process takes place on all the computational nodes. 


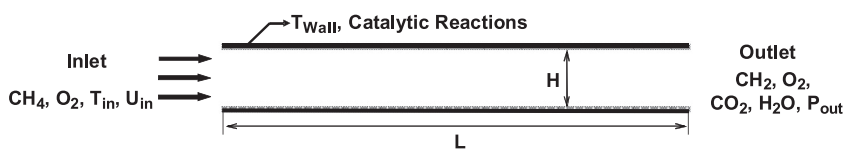

FIG. 7. Catalytic reaction in a channel flow with temperature gradients. Premixed methane-air mixtures enter a channel coated with platinum. The channel has a prescribed constant wall temperature, length $L$, and height $H$.

$\rho_{j w}$ the corresponding density. The mass fluxes can also be written in terms of populations as

$$
\begin{aligned}
& \dot{\rho}_{j}^{\text {in }}=\sum_{i}^{c_{j i \alpha} n_{\alpha}>0}\left|f_{j i} c_{j i \alpha} n_{\alpha}\right|, \\
& \dot{\rho}_{j}^{\text {out }}=\sum_{i}^{c_{j i \alpha} n_{\alpha}<0}\left|f_{j i} c_{j i \alpha} n_{\alpha}\right|,
\end{aligned}
$$

where $n_{\alpha}$ denotes inward direction normal to the wall. For the diffusive $\mathrm{BC}$, the incoming populations $f_{j i}^{\mathrm{eq}}$ can be expressed as

$$
f_{j i}^{\mathrm{in}}=f_{j i}^{\mathrm{eq}}\left(\rho_{j w}, U_{w}, T_{w}\right) \frac{\dot{\rho}_{j}^{\mathrm{in}}}{\dot{\rho}_{j i}^{\mathrm{eq}, \mathrm{in}}\left(\rho_{j w}, U_{w}, T_{w}\right)},
$$

where $f_{j i}^{\mathrm{eq}}\left(\rho_{j w}, U_{w}, T_{w}\right)$ are the equilibrium populations calculated with the density $\rho_{j w}$, the wall velocity $U_{w}$, and the wall temperature $T_{w}$. The denominator in Eq. (50) indicates the incoming flux calculated with equilibrium populations $f_{j i}^{\mathrm{eq}}\left(\rho_{j w}, U_{w}, T_{w}\right)$.

Substituting Eqs. (49) and (48), Eq. (50) becomes

$$
f_{j i}^{\mathrm{in}}=f_{j i}^{\mathrm{eq}}\left(\rho_{j}, U_{w}, T_{w}\right) \frac{\sum_{i}^{c_{j i \alpha} n_{\alpha}<0}\left|f_{j i} c_{j i \alpha} n_{\alpha}\right|+M_{j} \dot{\omega}_{j}}{\sum_{i}^{c_{j i \alpha} n_{\alpha}>0}\left|f_{j i}^{\mathrm{eq}}\left(\rho_{j}, U_{w}, T_{w}\right) c_{j i \alpha} n_{\alpha}\right|} .
$$

It is seen that the density of each species at the wall, $\rho_{j w}$, which is unknown a priori, is needed in Eq. (48) for evaluating $\dot{\omega}_{j}$ and in Eq. (50) for calculating equilibrium populations. There is more than one way to obtain $\rho_{j w}[21,50]$. Here we select to take as initial guess for $\rho_{j w}$ the density value on its direct neighboring node in the fluid, and to update $\rho_{j w}$ iteratively by using properties of the zeroth-order moment of the distribution functions:

$$
\rho_{j w}=\sum_{i}^{0, \ldots, 8} f_{j i}=\sum_{i}^{c_{j i \alpha} n_{\alpha}>0} f_{j i}+\sum_{i}^{c_{j i \alpha} n_{\alpha}<0} f_{j i}+\sum_{i}^{c_{j i \alpha} n_{\alpha}=0} f_{j i} .
$$

TABLE III. Conditions for two cases of surface reactive flows in heated catalytic channel.

\begin{tabular}{lrrrrr}
\hline \hline $\begin{array}{l}\text { Simulation } \\
\text { cases }\end{array}$ & $\begin{array}{c}U_{\text {in }}^{\mathrm{a}} \\
(\mathrm{m} / \mathrm{s})\end{array}$ & $\begin{array}{c}T_{\text {in }} \\
(\mathrm{K})\end{array}$ & $\begin{array}{c}T_{\text {wall }} \\
(\mathrm{K})\end{array}$ & \multicolumn{2}{c}{ Mole fraction } \\
\hline I & 7.2 & 1200 & 1200 & 10 & 90 \\
II & 11.5 & 400 & 1200 & 10 & 90 \\
\hline \hline
\end{tabular}

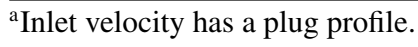

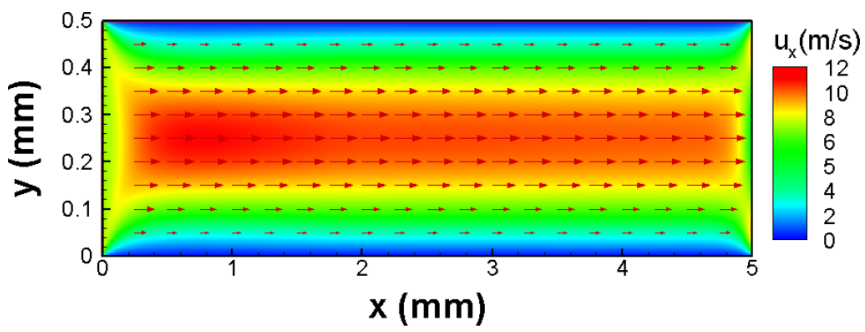

FIG. 8. (Color online) Catalytic channel flow: Case I: Velocity vector field and contour map of the streamwise velocity field.

Substituting Eqs. (48) and (49), we obtain

$$
\rho_{j w}=2 \sum_{i}^{c_{j i \alpha} n_{\alpha}<0} f_{j i}+\sum_{i}^{c_{j i \alpha} n_{\alpha}=0} f_{j i}+M_{j} \dot{\omega}_{j} .
$$

For better understanding, the algorithm for the computation of the unknown density $\rho_{j w}$ and incoming distribution function $f_{j i}^{\text {in }}$ at the wall is presented schematically in Fig. 5. The example of a catalytic wall is used (see Fig. 6). In the current scheme, the wall is placed on the grid at $y=0$. One can also locate the wall at the halfway position between the lattice grid points for increased accuracy. This would involve slightly more computations, and for presentation purposes we select to work with the wall placed on the grid. In the implementation of the current boundary condition, first, the density on the neighboring point $y=1$ is used as initial guess for the density at the boundary $y=0$, leading to an approximation of the production rate $\dot{\omega}_{j}$. Thereafter, the inward mass flux $\dot{\rho}_{j}^{\text {in }}$ is calculated by using Eq. (48), and the density at the wall $\rho_{j w}^{*}$ is obtained by using Eq. (53). By comparing the new $\rho_{j w}^{*}$ with the initially assumed $\rho_{j w}$, it is assessed whether the computation of $\rho_{j w}$ is correct or more iterations are necessary. When converged, the equilibrium distribution at the wall can be calculated with the prescribed properties, $U_{w}, T_{w}$ and the obtained density $\rho_{j w}$. Finally, the incoming populations $f_{j i}^{\text {in }}$ are calculated by Eq. (51). The results of the density and populations are stored, and the computation proceeds with the LB propagation and collision calculation for this time step.

\section{BENCHMARKING: CATALYTIC REACTIVE FLOW IN A HEATED CHANNEL}

\section{A. Problem description}

In this section the proposed thermal multicomponent model together with the catalytic boundary condition of the previous

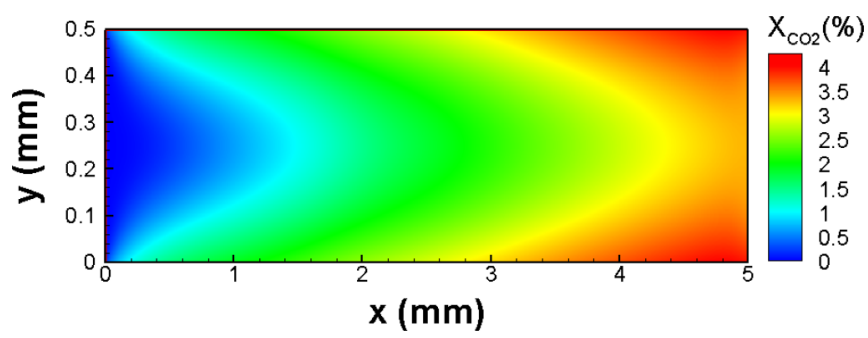

FIG. 9. (Color online) Catalytic reaction channel flow: Case I: Contour map of $\mathrm{CO}_{2}$ mole fraction. 


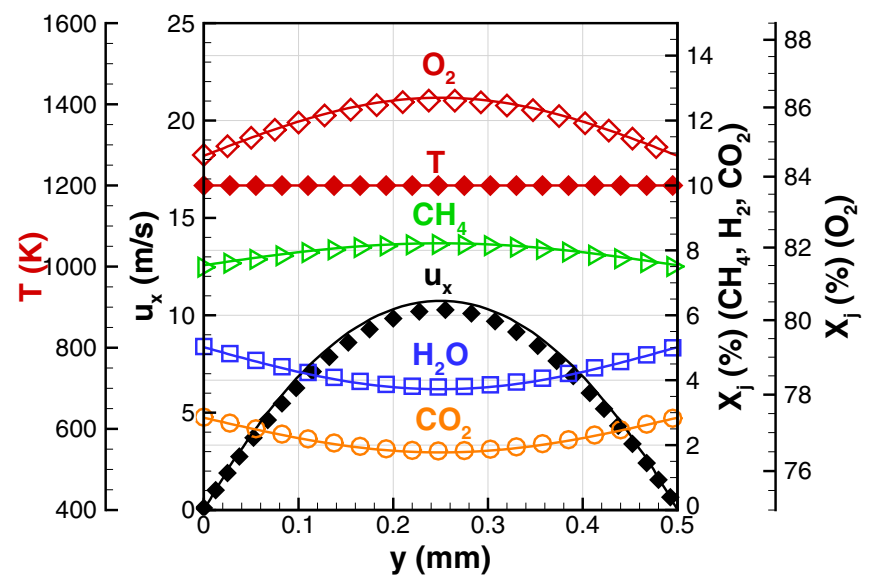

FIG. 10. (Color online) Catalytic reaction channel flow: Case I: Comparison between the results of LB model (symbols) and the finite volume solver [27] (solid lines) along the cross section in the middle of the channel. The red line and adjacent filled diamonds represent the temperature, the black line and adjacent filled diamonds the streamwise velocity, the green line and adjacent triangles the $\mathrm{CH}_{4}$ mole fraction, the blue line and adjacent open squares the $\mathrm{H}_{2} \mathrm{O}$ mole fraction, the red line and adjacent open diamonds the $\mathrm{O}_{2}$ mole fraction, and the orange line and adjacent open circles the $\mathrm{CO}_{2}$ mole fraction.

section are applied to simulate the reactive flow in a catalytic channel under large temperature gradients. As shown in Fig. 7, a premixed methane-air mixture enters a planar catalytic channel with inlet temperature $T_{\text {in }}$ and velocity $U_{\text {in. }}$. The channel is $5 \mathrm{~mm}$ long and $0.5 \mathrm{~mm}$ high and is kept at a constant wall temperature $T_{\text {wall }}$. The mixture flow is heated up when it passes through the channel. Simultaneously, the catalytic oxidation of methane occurs at the catalytic walls. The surface chemistry is treated as one-step global reaction for the catalytic total oxidation of methane on platinum:

$$
\mathrm{CH}_{4}+2 \mathrm{O}_{2} \rightarrow 2 \mathrm{H}_{2} \mathrm{O}+\mathrm{CO}_{2}, \quad k_{r}=A \exp \left(-E_{a} / R T_{w}\right),
$$

where $k_{r}$ is the global reaction rate constant, $A=1.27 \times$ $10^{5} \mathrm{~cm} / \mathrm{s}, E_{a}=77 \mathrm{KJ} / \mathrm{mol}$ [26] is the apparent activation energy, $T_{\text {wall }}$ is the temperature of the top and bottom channel walls. The production rate $\dot{\omega}_{\mathrm{CH} 4}$ of methane under lean stoichiometries is a function of methane concentration with a first-order dependence, $\dot{\omega}_{\mathrm{CH} 4}=-k_{r}\left[\mathrm{CH}_{4}\right]$, which in turn gives the production/destruction rates of the other species as $\dot{\omega}_{\mathrm{O} 2}=2 \dot{\omega}_{\mathrm{CH} 4}, \dot{\omega}_{\mathrm{H} 2 \mathrm{O}}=-2 \dot{\omega}_{\mathrm{CH} 4}$, and $\dot{\omega}_{\mathrm{CO} 2}=-\dot{\omega}_{\mathrm{CH} 4}$.

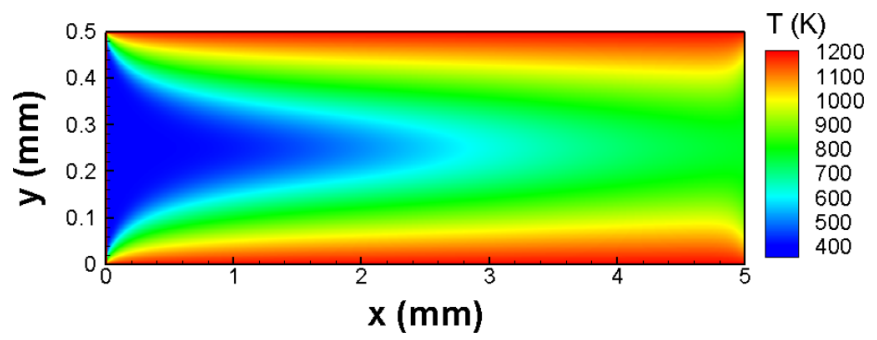

FIG. 11. (Color online) Catalytic reaction channel flow: Case II: Contour map for the temperature field.

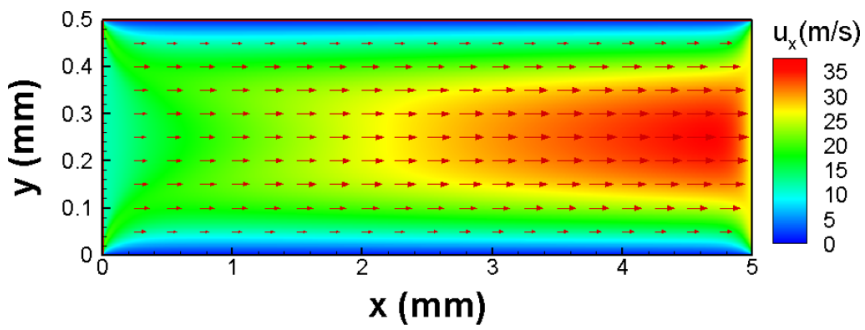

FIG. 12. (Color online) Catalytic reaction channel flow: Case II: Velocity vector field and contour map of the streamwise velocity field.

Production rates, as well as transport and thermodynamic properties of each species, are dynamically evaluated from the CHEMKIN package $[39,46]$ at every time step. The transformation from physical units to LB units is explained in Appendix C. Under different inflow conditions, i.e., inlet velocity and inlet temperature, the LB simulation results are compared against the finite volume Navier-Stokes solver at PSI [27].

\section{B. Simulation conditions and results}

Two simulations under different conditions are presented. Table III provides values of the inlet velocity, temperature, and composition. Case I is a simpler configuration, with no temperature gradients. For case II, the difference between the inlet flow temperature and wall temperature is set such that it creates steep gradients.

In the simulation, the inlet boundary is implemented by setting populations at equilibrium. The equilibrium populations are calculated using the inlet temperature and mole fraction given in Table III, the total density and velocity of the neighboring inner node. The nodes at the top and bottom corners of inlet are treated as solid stationary wall nodes by imposing an equilibrium population calculated at zero velocity and wall temperature and with the density of neighboring wall nodes (no catalytic reaction is considered). The outlet boundary is imposed with equilibrium populations, which are evaluated with the species velocity, $u_{j \alpha}$, mole fraction, $X_{j}$, and temperature, $T$, evaluated on the neighboring fluid nodes in the inner domain, and the density calculated with desired pressure $P_{\text {out }}$ (in both cases, $P_{\text {out }}=1$ bar), $\rho=\bar{M} P_{\text {out }} / T$.

The simulation domain is discretized by $N_{x} \times N_{y}=$ $2000 \times 200$ grid points along the horizontal $x$ and vertical $y$ direction, respectively. For the simulation of case I, the LB results are shown in Figs. 8-10. Figure 8 presents the velocity

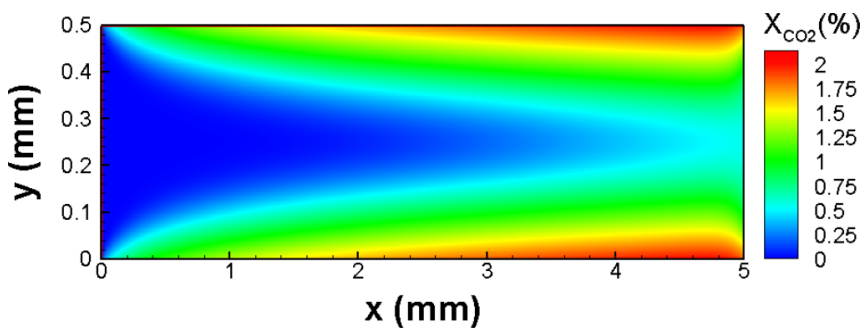

FIG. 13. (Color online) Catalytic reaction channel flow: Case II: Contour map of $\mathrm{CO}_{2}$ mole fraction. 


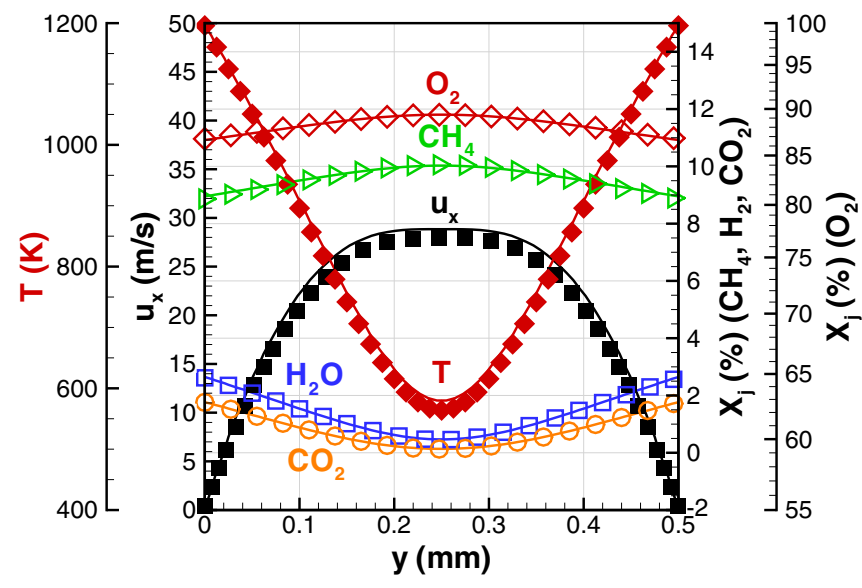

FIG. 14. (Color online) Catalytic reaction channel flow: Case II: Comparison between the results of LB model (symbols) and the finite volume solver [27] (solid lines) along the cross section in the middle of the channel. The red line and adjacent filled diamonds represent the temperature, the black line and adjacent filled squares the streamwise velocity, the green line and adjacent triangles the $\mathrm{CH}_{4}$ mole fraction, the blue line and adjacent open squares the $\mathrm{H}_{2} \mathrm{O}$ mole fraction, the red line and adjacent open diamonds the $\mathrm{O}_{2}$ mole fraction, and the orange line and adjacent open circles the $\mathrm{CO}_{2}$ mole fraction.

vector field and the contour map of the streamwise velocity. Figure 9 shows the mole fraction of the product $\mathrm{CO}_{2}$. Due to the surface catalytic reaction, methane and oxygen are consumed, and the concentration of $\mathrm{CO}_{2}$ is increasing gradually along the channel. In Fig. 10 the LB results (symbols) are compared against a finite volume solver [27] (solid lines). The curves and symbols show the profiles along the transverse cross section in the middle of the channel $(x=2.5 \mathrm{~mm})$. The bending of the concentration profiles near the wall clearly demonstrates the depletion of the reactants and the formation of products. The results obtained by the two methods are in good agreement.

For the simulation of case II, the obtained temperature field, velocity field and mole fraction of the product $\mathrm{CO}_{2}$ are shown in Figs. 11, 12, and 13, respectively. The results demonstrate that when the $400 \mathrm{~K}$ methane-air mixture passes through the hot channel, the gaseous mixture is heated up, the volume is expanded, and the flow is accelerated. At the same time, the mole fraction of product $\mathrm{CO}_{2}\left(X_{\mathrm{CO} 2}\right.$ in Fig. 13) gradually increases along the streamwise direction, indicating a catalytic reaction on the channel wall. In Fig. 14 LB profiles (symbols) and predictions from the finite volume solver (solid lines) are compared along the cross section in the middle of the channel. The comparison shows that, while large temperature gradients exist in the channel flow, the LB results are in good agreement with the predictions of the finite volume solver.

\section{CONCLUSION}

In this paper the kinetic model for thermal binary-mixture flows proposed in Ref. [18] was extended in order to describe thermal multicomponent flows. The macroscopic limits of the bare LB model have been studied and the resulting deviation was identified via the Chapman-Enskog analysis. The deviation was neutralized by introducing exact correction terms at the level of the lattice BGK equation, thus guaranteeing the correct thermo-hydrodynamic limits. The resulting relations between the relaxation times and the transport properties have been identified. The values of viscosity, diffusivity, and conductivity can now be independently applied for each species allowing a more realistic description of mixture flows. Moreover, this allows the integration of the CHEMKIN package and the dynamic evaluation of the physical properties of each species.

The compressibility and physical integrity of the model was studied by simulating the speed of sound in gaseous mixtures of different compositions at various temperatures. The agreement between LB simulation and the theoretical values indicates the potential of the proposed model to study acoustic waves in combustion processes.

The distinct features of the proposed model were further demonstrated through the simulation of catalytic reactive thermal flows. For this purpose, the diffusive boundary condition was adapted to include the temperature effects in addition to the surface chemistry. The thermal multicomponent model together with the thermal catalytic boundary scheme were applied to simulate heterogeneous reactive flows under large temperature variation. The LB simulation results were in good agreement with a finite volume Navier-Stokes Elliptic solver.

\section{ACKNOWLEDGMENTS}

We gratefully acknowledge the support from the Swiss Federal Office of Energy (BFE) via projects 103078 and 153708 and from the European Union HRC-Power project.

\section{APPENDIX A: EQUILIBRIUM HIGHER ORDER MOMENTS AND THEIR DEVIATIONS}

The moments of the equilibrium populations are by construction equal to the ones predicted by the kinetic theory up to the second order. The form of the second- and higher order moments of species $j(j=1,2, \ldots, N$, is not a summation index below) are

$$
\begin{aligned}
P_{j \alpha \beta}^{\mathrm{eq}}= & P_{j \alpha \beta}^{\mathrm{MB}}=C_{j} T \delta_{\alpha \beta}+\frac{\rho_{j} J_{\alpha} J_{\beta}}{\rho^{2}}, \\
Q_{j \alpha \beta \gamma}^{\mathrm{eq}}= & Q_{j \alpha \beta \gamma}^{\mathrm{MB}}+Q_{j \alpha \beta \gamma}^{\prime} \\
= & C_{j} T\left(\frac{J_{\alpha}}{\rho} \delta_{\beta \gamma}+\frac{J_{\beta}}{\rho} \delta_{\alpha \gamma}+\frac{J_{\gamma}}{\rho} \delta_{\alpha \beta}\right) \\
& +\frac{\rho_{j} J_{\alpha} J_{\beta} J_{\gamma}}{\rho^{3}}+Q_{j \alpha \beta \gamma}^{\prime}, \\
q_{j \alpha}^{\mathrm{eq}}= & q_{j \alpha}^{\mathrm{MB}}+q_{j \alpha}^{\prime}=Y_{j} J_{\alpha}\left(\frac{J^{2}}{\rho^{2}}+\frac{4 T}{M_{j}}\right)+q_{j \alpha}^{\prime}, \\
R_{j \alpha \beta}^{\mathrm{eq}}= & R_{j \alpha \beta}^{\mathrm{MB}}+R_{j \alpha \beta}^{\prime} \\
= & C_{j} R T\left(\frac{J^{2}}{\rho^{2}}+\frac{4 T}{M_{j}}\right) \delta_{\alpha \beta} \\
& +\frac{\rho_{j} J_{\alpha} J_{\beta}}{\rho^{2}}\left(\frac{6 T}{M_{j}}+\frac{J^{2}}{\rho^{2}}\right)+R_{j \alpha \beta}^{\prime},
\end{aligned}
$$


where $Q_{j \alpha \beta \gamma}^{\prime}, q_{j \alpha}^{\prime}$, and $R_{j \alpha \beta}^{\prime}$ are the deviations of the higher order moments from the Maxwell-Boltzmann (MB) form. These deviations are calculated exactly:

$$
\begin{aligned}
q_{j \alpha}^{\prime} & =\frac{1-3 T}{M_{j}} Y_{j} J_{\alpha}-\frac{Y_{j} J_{\alpha}^{3}}{\rho^{2}}, \quad Q_{j x x y}^{\prime}=Q_{j x y y}^{\prime}=0, \quad Q_{j x x x}^{\prime}=\frac{1-3 T}{M_{j}} Y_{j} J_{x}-\frac{Y_{j} J_{x}^{3}}{\rho^{2}}, \quad Q_{j y y y}^{\prime}=\frac{1-3 T}{M_{j}} Y_{j} J_{y}-\frac{Y_{j} J_{y}^{3}}{\rho^{2}}, \\
R_{j x y}^{\prime} & =(2-6 T) \frac{C_{j} J_{x} J_{y}}{\rho^{2}}-\frac{Y_{j} J_{x} J_{y} J^{2}}{\rho^{3}}, \quad R_{j x x}^{\prime}=\frac{1-3 T}{M_{j}} C_{j} T+\frac{(1-6 T) C_{j} J_{x}^{2}}{\rho^{2}}+\frac{Y_{j} J_{x}^{4}}{\rho^{3}}, \\
R_{j y y}^{\prime} & =\frac{1-3 T}{M_{j}} C_{j} T+\frac{(1-6 T) C_{j} J_{y}^{2}}{\rho^{2}}+\frac{Y_{j} J_{y}^{4}}{\rho^{3}} .
\end{aligned}
$$

\section{APPENDIX B: CORRECTION TERMS}

\section{Momentum equation}

The deviation term that exists in the momentum equation Eq. (29) of the bare LB model, i.e., before applying the correction mechanism, is $\partial_{\beta} P_{\alpha \beta}^{\prime \prime}=\sum_{j}^{N} \partial_{\beta} P_{j \alpha \beta}^{\prime \prime}$. The exact form is identified through Chapman-Enskog analysis:

$$
\begin{aligned}
\partial_{\beta} P_{j x \beta}^{\prime \prime}= & -\partial_{x}\left\{\begin{array}{l}
\tau_{j 1} \partial_{x}\left(\frac{1-3 T}{M_{j}} Y_{j} J_{x}-\frac{Y_{j} J_{x}^{3}}{\rho^{2}}\right) \\
-\frac{\tau_{j 1} X_{j}}{D}\left[\partial_{x}\left(\frac{1-3 T}{\bar{M}} J_{x}-\frac{J_{x}^{3}}{\rho^{2}}\right)+\partial_{y}\left(\frac{1-3 T}{\bar{M}} J_{y}-\frac{J_{y}^{3}}{\rho^{2}}\right)\right]
\end{array}\right\} \\
& +\left\{\begin{array}{l}
2 \partial_{x}\left[\frac{\tau_{j 2} J_{x}}{\rho}\left(Y_{j} \partial_{x} p-\partial_{x} p_{j}\right)\right] \\
+\partial_{y}\left[\frac{\tau_{j 2} J_{x}}{\rho}\left(Y_{j} \partial_{y} p-\partial_{y} p_{j}\right)+\frac{\tau_{j 2} J_{y}}{\rho}\left(Y_{j} \partial_{x} p-\partial_{x} p_{j}\right)\right]
\end{array}\right\}, \\
\partial_{\beta} P_{j y \beta}^{\prime \prime}= & -\partial_{y}\left\{\begin{array}{l}
\tau_{j 1} \partial_{y}\left(\frac{1-3 T}{M_{j}} Y_{j} J_{y}-\frac{Y_{j} J_{y}^{3}}{\rho^{2}}\right) \\
-\frac{\tau_{j 1} X_{j}}{D}\left[\partial_{x}\left(\frac{1-3 T}{\bar{M}} J_{x}-\frac{J_{x}^{3}}{\rho^{2}}\right)+\partial_{y}\left(\frac{1-3 T}{\bar{M}} J_{y}-\frac{J_{y}^{3}}{\rho^{2}}\right)\right]
\end{array}\right\} \\
& +\left\{\begin{array}{l}
2 \partial_{y}\left[\frac{\tau_{j 2} J_{y}}{\rho}\left(Y_{j} \partial_{y} p-\partial_{y} p_{j}\right)\right] \\
+\partial_{x}\left[\frac{\tau_{j 2} J_{y}}{\rho}\left(Y_{j} \partial_{x} p-\partial_{x} p_{j}\right)+\frac{\tau_{j 2} J_{x}}{\rho}\left(Y_{j} \partial_{y} p-\partial_{y} p_{j}\right)\right]
\end{array}\right\} .
\end{aligned}
$$

This deviation is eliminated using the counterterm $\psi_{j i}^{(\text {II) }}$. Note that the second row of bracketed terms in Eqs. (B1a)-(B1b) does not exist in the case of the thermal binary mixture of Ref. [18].

\section{Energy equation}

The deviation term in energy equation (37) of the bare LB model is expressed in two parts, $\partial_{\alpha} q_{\alpha}^{\prime}$ and $\partial_{\alpha} q_{\alpha}^{\prime \prime}$. The first part originates from the deviation of the equilibrium part of the third order contracted moment, which is directly given by Eq. (A2). The second part, $\partial_{\alpha} q_{\alpha}^{\prime \prime}=\sum_{j}^{N} \partial_{\alpha} q_{j \alpha}^{\prime \prime}$, stems from the deviation of the nonequilibrium part of the third-order contracted moment, and was identified through Chapman-Enskog analysis. The exact form of $\partial_{\alpha} q_{j \alpha}^{\prime \prime}$ for species $j$ is

$$
q_{j \alpha}^{\prime \prime}=+\frac{3 \tau_{j 1} C_{j} T}{M_{j}} \partial_{\alpha} T+\frac{\tau_{j 2}(1-3 T)}{M_{j}}\left[Y_{j} \partial_{\alpha} p-\partial_{\alpha} p_{j}\right]+\tau_{j 1}\left\{\begin{array}{l}
-\frac{3 Y_{j} J_{\alpha}^{2}}{\rho^{2}} \partial_{\alpha} p-\frac{3 X_{j} p J_{\alpha}}{\rho} \partial_{\beta} \frac{J_{\beta}}{\rho}+\frac{3 X_{j} C J_{\alpha} J_{\beta}}{2 \rho^{2}} \partial_{\beta} T \\
-(1-3 T) \partial_{\beta} \frac{X_{j} C J_{\alpha} J_{\beta}}{\rho^{2}}+\frac{X_{j}(1-3 T) J_{\alpha}}{2 \rho} \partial_{\beta} \frac{J_{\beta}}{\bar{M}} \\
+\partial_{\alpha} \frac{C_{j} J_{\alpha}^{2}}{\rho^{2}}+\left(\frac{1}{\tau_{j 1}}-\frac{1}{\tau_{j 2}}\right)\left(\frac{J_{j \alpha} J_{j}^{2}}{\rho_{j}^{2}}-\frac{Y_{j} J_{\alpha} J^{2}}{\rho^{2}}\right) \\
-\partial_{\beta} \frac{Y_{j} J_{\beta} J_{\alpha}^{3}}{\rho^{3}}-\frac{X_{j} J_{\alpha}}{2 \rho} \partial_{\beta} \frac{J_{\beta}^{3}}{\rho^{2}}-\partial_{\alpha} \frac{Y_{j} J_{\alpha}^{4}}{\rho^{3}}+\partial_{\beta} \frac{Y_{j} J_{\alpha} J_{\beta} J^{2}}{\rho^{3}}
\end{array}\right\},
$$

where $\alpha$ indicates the direction ( $x$ or $y$ ), and Einstein summation is assumed only for the repeated index $\beta$. Similar to the momentum equation deviations [Eqs. (B1a)-(B1b)], the deviation of the energy equation of the thermal multicomponent mixture is different compared to the thermal binary mixture of Ref. [18].

In usual flow cases, many of the deviation terms can be neglected, simplifying the scheme. However, it is worth stressing that for a fully Galilean invariant scheme, all these deviation terms must be neutralized.

\section{APPENDIX C: TRANSFORMATION OF UNITS FOR THE CATALYTIC SURFACE REACTION MECHANISM}

In order to integrate CHEMKIN libraries into the LB algorithm, the units should be transformed from physical to 
LB ones. The transformation follows a standard procedure. First, length scale $L^{\prime}$ is chosen, which is the relation between the characteristic length of the physical system $L$ and the discretized lattice characteristic length $L_{\mathrm{LB}}$ (number of grid points):

$$
L^{\prime}=\frac{L}{L_{\mathrm{LB}}} .
$$

The next step is to choose the velocity scale $U^{\prime}$. The principle used in Ref. [38] is applied: $U^{\prime}$ is defined such that after the velocities are scaled into LB units, the lattice velocity of the lightest species $M_{0, \mathrm{LB}}=1$ of the mixture is equal to $c_{0, \mathrm{LB}}=1$. For the D2Q9 lattice implementation, this velocity scale is

$$
U^{\prime}=\sqrt{\frac{3 T_{0}}{M_{0}}},
$$

where $T_{0}$ is reference temperature in physical units, $M_{0}$ is the molecular weight of the lightest species in physical units.
Since the length scale and velocity scales are known, the time scale is simply obtained by

$$
t^{\prime}=\frac{L^{\prime}}{U^{\prime}}
$$

The density scale is set to $\rho^{\prime}=1 \mathrm{~kg} / \mathrm{m}^{3}$, making the physical density and LB density equal, $\rho=\rho_{\mathrm{LB}}$. The temperature scale is defined by selecting a physical reference temperature $T_{0}$ and the reference temperature in LB units $T_{0, \mathrm{LB}}$ :

$$
T^{\prime}=\frac{T_{0}}{T_{0, \mathrm{LB}}},
$$

where $T_{0, \mathrm{LB}}$ is calculated with Eq. (C2) by substituting the velocity with $c_{0, \mathrm{LB}}$ and the molecular weight with $M_{0, \mathrm{LB}}$.

As an example, the physical reference temperature $T_{0}$ in the simulation of Sec. VI was set to $T_{0}=1200 \mathrm{~K}$. Based on above scaling factors, the variables with physical units and LB units are calculated in CHEMKIN and LB solvers and transferred to each other.
[1] S. Succi, E. Foti, and F. Higuera, Europhys. Lett. 10, 433 (1989).

[2] R. Benzi, S. Succi, and M. Vergassola, Phys. Rep. 222, 145 (1992).

[3] Y. H. Qian, D. d'Humieres, and P. Lallemand, Europhys. Lett. 17, 479 (1992).

[4] X. Shan and H. Chen, Phys. Rev. E 47, 1815 (1993).

[5] S. Chen and G. Doolen, Annu. Rev. Fluid Mech. 30, 329 (1998).

[6] S. Succi, The Lattice Boltzmann Equation for Fluid Dynamics and Beyond (Oxford University Press, Oxford, 2001).

[7] Q. Li and A. J. Wagner, Phys. Rev. E 76, 036701 (2007).

[8] R. Benzi, M. Sbragaglia, S. Succi, M. Bernaschi, and S. Chibbaro, J. Chem. Phys. 131, 104903 (2009).

[9] T. Rosén, J. Eller, J. Kang, N. I. Prasianakis, J. Mantzaras, and F. N. Büchi, J. Electrochem. Soc. 159, F536 (2012).

[10] X. Shan and G. Doolen, Phys. Rev. E 54, 3614 (1996).

[11] L.-S. Luo and S. S. Girimaji, Phys. Rev. E 66, 035301 (2002).

[12] L.-S. Luo and S. S. Girimaji, Phys. Rev. E 67, 036302 (2003).

[13] M. E. McCracken and J. Abraham, Phys. Rev. E 71, 046704 (2005).

[14] P. Asinari, Physics of Fluids 17, 067102 (2005).

[15] S. Arcidiacono, S. Ansumali, I. V. Karlin, J. Mantzaras, and K. B. Boulouchos, Math. Comput. Simul. 72, 79 (2006).

[16] S. Arcidiacono, J. Mantzaras, S. Ansumali, I. V. Karlin, C. Frouzakis, and K. B. Boulouchos, Phys. Rev. E 74, 056707 (2006).

[17] P. Asinari, Phys. Rev. E 73, 056705 (2006).

[18] J. Kang, N. I. Prasianakis, and J. Mantzaras, Phys. Rev. E. 87, 053304 (2013).

[19] X. Shan, Phys. Rev. E 81, 045701 (2010).

[20] J. Ross, Heterogeneous Catalysis: Fundamentals and Applications (Elsevier, New York, 2011).

[21] S. Arcidiacono, J. Mantzaras, and I. V. Karlin, Phys. Rev. E 78, 046711 (2008).

[22] C. Bohn, S. Scott, J. Dennis, and C. Müller, J. Comp. Phys. 231, 5334 (2012).

[23] G. Prisco and X. Shan, Commun. Comput. Phys. 9, 1362 (2011).
[24] M. Kamali, S. Sundaresan, H. Van den Akker, and J. Gillissen, Chem. Eng. J. 207-208, 587 (2012).

[25] S. Ansumali and I. V. Karlin, Phys. Rev. E 66, 026311 (2002).

[26] M. Reinke, J. Mantzaras, R. Schaeren, R. Bombach, A. Inauen, and S. Schenker, Combust. Flame 136, 217 (2004).

[27] Y. Ghermay, J. Mantzaras, and R. Bombach, Combust. Flame 157, 1942 (2010).

[28] A. N. Gorban and I. V. Karlin, Physica A 206, 401 (1994).

[29] A. N. Gorban and I. V. Karlin, Invariant Manifolds for Physical and Chemical Kinetics (Springer, Berlin, 2005).

[30] C. D. Levermore, J. Stat. Phys. 83, 1021 (1996).

[31] N. I. Prasianakis and K. B. Boulouchos, Int. J. Mod. Phys. C 18, 602 (2007).

[32] I. V. Karlin, A. Ferrante, and H. C. Öttinger, Europhys. Lett. 47, 182 (1999).

[33] S. Ansumali and I. V. Karlin, Phys. Rev. Lett. 95, 260605 (2005).

[34] N. I. Prasianakis and I. V. Karlin, Phys. Rev. E 78, 016704 (2008).

[35] S. Chapman and T. G. Cowling, The Mathematical Theory of Non-Uniform Gases (Cambridge University Press, Cambridge, 1970).

[36] N. I. Prasianakis and I. V. Karlin, Phys. Rev. E 76, 016702 (2007).

[37] N. I. Prasianakis, I. V. Karlin, J. Mantzaras, and K. B. Boulouchos, Phys. Rev. E 79, 066702 (2009).

[38] S. Arcidiacono, I. V. Karlin, J. Mantzaras, and C. E. Frouzakis, Phys. Rev. E 76, 046703 (2007).

[39] R. Kee, G. Dixon-Lewis, J. Warnatz, M. Coltrin, and J. Miller, A Fortran Computer Code Package for the Evaluation of GasPhase Multicomponent Transport Properties (Sandia National Laboratories, Sandia, Albuquerque, NM, 1986).

[40] R. Bird, W. Stewart, and E. Lightfoot, Transport Phenomena (Wiley, New York, 1960).

[41] F. Williams, Combustion Theory (Benjamin/Cumming, Menlo Park, California, 1986). 
[42] P. Asinari, Phys. Rev. E 80, 056701 (2009).

[43] P. Asinari, Phys. Rev. E 77, 056706 (2008).

[44] P. Andries, K. Aoki, and B. Perthame, J. Stat. Phys. 106, 993 (2002).

[45] C. R. Wilke, J. Chem. Phys. 18, 517 (1950).

[46] M. Coltrin, R. Kee, and F. Rupley, Report no. SAND90-8003C, Sandia National Laboratories, Albuquerque, NM, 1996.
[47] S. Mathur, P. K. Tondon, and S. C. Saxena, Mol. Phys. 12, 569 (1967).

[48] V. Bellucci, C. Paschereit, B. Schuermans, D. Nowak, and P. Flohr, J. Turbomach. 127, 372 (2005).

[49] S. Ansumali, I. V. Karlin, C. E. Frouzakis, and K. B. Boulouchos, Physica A 359, 289 (2006).

[50] J. Kang, Ph.D. thesis, ETH-Zurich, 2013. 